Georgian Mathematical Journal

Volume 8 (2001), Number 4, 665-668

\title{
ON THE RATIONALITY OF CERTAIN STRATA OF THE LANGE STRATIFICATION OF STABLE VECTOR BUNDLES ON CURVES
}

\author{
E. BALLICO
}

\begin{abstract}
Let $X$ be a smooth projective curve of genus $g \geq 2$ and $S(r, d)$ the moduli scheme of all rank $r$ stable vector bundles of degree $d$ on $X$. Fix an integer $k$ with $0<k<r$. H. Lange introduced a natural stratification of $S(r, d)$ using the degree of a rank $k$ subbundle of any $E \in S(r, d)$ with maximal degree. Every non-dense stratum, say $W(k, r-k, a, d-a)$, has in a natural way a fiber structure $h: W(k, r-k, a, d-a) \rightarrow \operatorname{Pic}^{a}(X) \times \operatorname{Pic}^{b}(X)$ with $h$ dominant. Here we study the rationality or the unirationality of the generic fiber of $h$.
\end{abstract}

2000 Mathematics Subject Classification: 14H60.

Key words and phrases: Stable vector bundles on curves, moduli schemes of vector bundles, rational variety, unirational variety.

\section{INTRODUCTION}

Let $X$ be a smooth complete algebraic curve of genus $g \geq 2$ defined over an algebraically closed base field $K$ with $\operatorname{char}(K)=0$. Fix integers $r, d$ with $r \geq 1$ and $L \in \operatorname{Pic}(X)$. Let $S_{L}(r, d)$ be the moduli scheme of stable rank $r$ vector bundles on $X$ with determinant $L$ and $S(r, d)$ the moduli scheme of all stable rank $r$ vector bundles on $X$ with degree $d$. It is well-known ([14]) that $S(r, d)$ (resp. $\left.S_{L}(r, d)\right)$ is smooth, irreducible, of dimension $\left(r^{2}-1\right)(g-1)+g$ (resp. $\left.\left(r^{2}-1\right)(g-1)\right)$ and that $S_{L}(r, d)$ is unirational. The variety $S_{L}(r, d)$ is a fine moduli scheme if and only if $(r, d)=1$. P. E. Newstead ([11]) proved in many cases that $S_{L}(r, d)$ is rational. For other cases, see [1]. By [5] $S_{L}(r, d)$ is rational if $(r, d)=1$. In [6] H. Lange introduced the following stratification (called the Lange stratification) of the moduli scheme $S(r, d), r \geq 2$, depending on the choice of an integer $k$ with $0<k<r$. For any rank $r$ vector bundle E set $s_{k}(E):=k(\operatorname{deg}(E))-r(\operatorname{deg}(A))$, where $A$ is a rank $k$ subsheaf of $E$ with maximal degree. By [9] we have $s_{k}(E) \leq g k(r-k)$. If $E$ is stable, then $s_{k}(E)>0$. By [4], sect. 4, (see [8], Remark 3.14) for any $L$ and a general $E \in S_{L}(r, d)$ we have $s_{k}(E)=k(r-k)(g-1)+e$, where $e$ is the unique integer with $(r-1)(g-1) \leq e \leq(r-1) g$ and $e+k(r-k)(g-1) \equiv k d \bmod (r)$. For any integer $a$ set $V(k, r-k, a, d-a):=\left\{E \in S(r, d): s_{k}(E)=k d-r a\right\}$. This gives a stratification of $S(r, d)$ which will be called the Lange stratification of $S(r, d)$. Here we study the rationality or the unirationality of smaller strata 
of this stratification. Hence (setting $b=d-a$ ) we fix integers $r, k, a, b$ with $0<k<r$ and $a / r<b /(r-k)<a / r+g-1$. By [12], Th. 0.1, there is a non-empty open irreducible subset $W(k, r-a, a, b)$ of $V(k, r-k, a, b)$ such that every $E \in W(k, r-k, a, b)$ fits in an exact sequence

$$
0 \rightarrow H \rightarrow E \rightarrow Q \rightarrow 0
$$

with $H$ computing $s_{k}(E)$ (i.e. with $\operatorname{rank}(H)=k, \operatorname{deg}(H)=a, \operatorname{rank}(Q)=r-k$ and $\operatorname{deg}(Q)=b), H$ and $Q$ stable and such that $H$ is the only rank $k$ subsheaf of $E$ computing $s_{k}(E)$. This means that (up to a scalar) $E$ fits in a unique extension (1). Furthermore, varying $E$ in $W(k, r-k, a, a, b)$, the pairs $(H, Q)$ obtained in this way cover a Zariski dense constructible subset of $S(k, a) \times S(r-$ $k, b)$. Conversely, the generic extension of the generic element of $S(r-k, b)$ by the generic element of $S(k, a)$ is the generic element of $W(k, r-k, a, b)$. Hence there is a rational dominant map $W(k, r-k, a, b) \rightarrow \operatorname{Pic}^{a}(X) \times \operatorname{Pic}^{b}(X) \cong$ $\operatorname{Alb}(X) \times \operatorname{Alb}(X)$ sending $E$ into $(\operatorname{det}(H), \operatorname{det}(Q))$. For any $L \in \operatorname{Pic}^{a}(X)$ and any $M \in \operatorname{Pic}^{b}(X)$ set $W(k, r-k, a, b, L, M):=\{E \in W(k, r-k, a, b): E$ fits in a unique exact sequence $(1)$ and $L \cong \operatorname{det}(H)$ and $M \cong \operatorname{det}(Q)\}$. We are interested in the rationality or unirationality of the strata $W(k, r-k, a, b, L, M)$. In this paper we prove the following results.

Theorem 1. Fix integers $r, k, a, b$ with $0<k<r$ and $a / r<b /(r-k)<$ $a / r+g-1$. Then for a general pair $(L, M) \in \operatorname{Pic}^{a}(X) \times \operatorname{Pic}^{b}(X)$ the variety $W(k, r-k, a, b, L, M)$ is unirational.

Theorem 2. Fix integers $r, k, a, b$ with $0<k<r, a / r<b /(r-k)<$ $a / r+g-1,(k, a)=1$ and $(r-k, b)=1$. Then for a general pair $(L, M) \in$ $\operatorname{Pic}^{a}(X) \times \operatorname{Pic}^{b}(X)$ the variety $W(k, r-k, a, b, L, M)$ is rational.

\section{Proofs of Theorems 1 AND 2}

Lemma 1. Fix integers $u, v, a$ and $b$ with $u>0$ and $v>0$ and take $a$ general pair $(L, M) \in \operatorname{Pic}^{a}(X) \times \operatorname{Pic}^{b}(X)$. Then for a general pair $(A, B) \in$ $S_{L}(u, a) \times S_{M}(v, b)$ we have $h^{0}(X, \operatorname{Hom}(A, B))=\max \{0, b u-a v+u v(1-g)\}$ and $h^{1}(X, \operatorname{Hom}(A, B))=\max \{0,-b u+a v+u v(g-1)\}$.

Proof. Without the restrictions $\operatorname{det}(A) \cong L$ and $\operatorname{det}(B) \cong M$, this is a result of A. Hirschowitz (see [2], sect. 4, or [13], Th. 1.2, for a published proof). By semicontinuity and the openness of stability we obtain the result for a general pair $(L, M)$.

Lemma 2. Fix integers $u, v, a$ and $b$ with $u>0, v>0$ and $a / u<b / v$ and take a general pair $(L, M) \in \operatorname{Pic}^{a}(X) \times \operatorname{Pic}^{b}(X)$. Then for a general pair $(A, B) \in S_{L}(u, a) \times S_{M}(v, b)$ the general extension of $B$ by $A$ is stable.

Proof. Without the restrictions $\operatorname{det}(A) \cong L$ and $\operatorname{det}(B) \cong M$, this is proved in [13] during the proof of [13], Theorems 0.1 and 0.2. By the openness of stability we obtain the result for a general pair $(L, M)$. 
Lemma 3. Fix integers $u, v, a$ and $b$ with $u>0, v>0$ and $a / u<b / v<$ $a / u+g-1$. Take a general pair $(L, M) \in \operatorname{Pic}^{a}(X) \times \operatorname{Pic}^{b}(X)$. Then for a general pair $(A, B) \in S_{L}(u, a) \times S_{M}(v, b)$ the general extension, $E$, of $B$ by $A$ is stable, $s_{u}(E)=u b-v a$ and $A$ is the only rank u subbundle of $E$ computing $s_{u}(E)$.

Proof. Without the restrictions $\operatorname{det}(A) \cong L$ and $\operatorname{det}(B) \cong M$, this is [13], Th. 0.1 . By the openness of stability and the semicontinuity of the Lange invariant $s_{u}$ we obtain the result for a general pair $(L, M)$.

Now we can prove Theorems 2 and 1.

Proof of Theorem 2. The variety $S_{L}(k, a) \times S_{M}(r-k, b)$ is rational by [5], Th. 1.2. Since $(k, a)=(r-k, b)=1$, both $S_{L}(k, a)$ and $S_{M}(r-k, b)$ are fine moduli spaces and hence there is a universal family, $U$, of pairs $(A, B)$ of vector bundles on $S_{L}(k, a) \times S_{M}(r-k, b)$. For every $(A, B) \in S_{L}(k, a) \times S_{M}(r-k, b)$ we have $h^{0}(X, \operatorname{Hom}(A, B))=0$ because $\mu(B)=b /(r-k)>a / k=\mu(A)$ and both $A$ and $B$ are stable. Thus $h^{1}(X, \operatorname{Hom}(A, B))=k b-(r-k) a+k(r-k)(g-1)$ (Riemann-Roch), i.e. $h^{1}(X, \operatorname{Hom}(A, B))$ does not depend from the choice of the pair $(A, B) \in S_{L}(k, a) \times S_{M}(r-k, b)$ but only from the integers $k, r, a$ and $b$. Thus the vector spaces $H^{1}(X, \operatorname{Hom}(A, B)),(A, B) \in S_{L}(k, a) \times S_{M}(r-k, b)$, fit together to form a vector bundle $E X T$ on $S_{L}(k, a) \times S_{M}(r-k, b)$ : the relative Ext-functor considered in [7]; here we need the existence of $U$ (i.e. the conditions $(k, a)=(r-k, b)=1)$ for the construction of EXT. Since $E X T$ is a vector bundle over an irreducible rational variety, the total space of EXT is an irreducible rational variety. By [13], Th. 0.1, a non-empty open subset $V$ of EXT corresponds to elements of $W(k, r-k, a, b, L, M)$ and conversely a general element of $W(k, r-k, a, b, L, M)$ corresponds to a general element of EXT. Hence there is a rational dominant map, $f$, from EXT into $W(k, r-k, a, b, L, M)$. As explained in the introduction, the uniqueness part in [13], Th. 0.1, means that the rational map $f$ induces a generically bijective map from the projective bundle $P(E X T))$ onto $W(k, r-k, a, b, L ; M)$. Since $P(E X T))$ is rational and $\operatorname{char}(K)=0$, we conclude.

Proof of Theorem 1. Fix integers $x, y$ with $x>0, P \in X$ and $R \in \operatorname{Pic}^{y}(X)$. Since $S_{R}(x, y) \cong S_{R(u x P)}(x, y+u x)$ for every integer $u$, we will assume $y$ very large, say $y>x(2 g-1)$. By the very construction of $S_{R}(x, y), y>x(2 g-1)$, using Geometric Invariant Theory, there is a smooth variety $U_{R}(x, y)$ with a PGL(N)-action, $N=y+x(1-g)$, without any fixed point and a morphism $f_{x, y}$ : $U_{R}(x, y) \rightarrow S_{R}(x, y)$ which make $S_{R}(x, y)$ the GIT-quotient of $U_{R}(x, y)$ and such that on $U_{R}(x, y) \times X$ there exists a total family of vector bundles on $X$ with $R$ as determinant. We repeat the proof of Theorem 2 using $U_{L}(k, a) \times U_{M}(r-k, b)$ instead of $S_{L}(k, a) \times S_{M}(r-k, b)$. Since on $U_{L}(k, a) \times U_{M}(r-k, b)$ there is a family of pairs of stable vector bundles, we may take a global EXT which is a vector bundle over $U_{L}(k, a) \times U_{M}(r-k, b)$ and hence it is irreducible and rational. By [13], Th. 0.1, there are a non-empty open subset $V$ of EXT and a 
dominant morphism $f: V \rightarrow W(k, r-k, a, b, L, M)$. Thus $W(k, r-k, a, b, L, M)$ is unirational.

\section{ACKNOWLEDGEMENT}

The author was partially supported by MURST (Italy).

\section{REFERENCES}

1. H. Boden and K. Yokogawa, Rationality of moduli spaces of parabolic bundles. $J$. London Math. Soc. 59(1999), 461-478.

2. L. Brambila-PaZ and H. Lange, A stratification of the moduli space of vector bundles on curves. J. Reine Angew. Math. 494(1998), 173-187.

3. R. Hernandez, Appendix to 'On regular and stable surfaces in $\mathbf{P}^{3}$. Algebraic Curves and Projective Geometry, Proceedings, Trento 1998, 16-17, Lect. Notes in Math. 1389, Springer-Verlag, Berlin / Heidelberg / New York, 1989.

4. A. Hirschowitz, Problémes de Brill-Noether en rang supérieur. Prepublication Mathematiques No. 91, Nice, 1986.

5. A. King and A. Schofield, Rationality of moduli of vector bundles on curves. Indag. Math. (N.S.) 10(1999), 519-535.

6. H. Lange, Zur Klassification von Regelmannigfaltigkeiten. Math. Ann. 262(1983), 447459.

7. H. Lange, Universal families of extensions. J. Algebra 83(1983), 101-112.

8. H. Lange, Some geometrical aspects of vector bundles on curves. L. Brambila Paz (ed.) et. al., Topics on algebraic geometry. Proceedings of a seminar on algebraic geometry, Guanajuato, Mexico, 1989. Mexico City: Sociedad Matematica Mexicana, Aportaciones Mat., Notas Invest. 5(1992), 53-74.

9. S. Mukai and F. SAKAI, Maximal subbundles of vector bundles on curves. Manuscripta Math. 52(1985), 251-256.

10. D. Mumford and P. E. Newstead, Periods of a moduli space of bundles on curves. Amer. J. Math. 90(1968), 1200-1208.

11. P. E. Newstead, Rationality of moduli spaces of stable bundles. Math. Ann. 215(1975), 251-268; Correction: 249(1980), 281-282.

12. S. Ramanan, The moduli space of vector bundles over an algebraic curve. Math. Ann. 200(1973), 69-84.

13. B. Russo and M. Teixidor i Bigas, On a conjecture of Lange. J. Algebraic Geometry 8(1999), 483-496.

14. C. S. Seshadri, Fibrés vectoriels sur les courbes algébriques. Astérisque 96(1992).

(Received 22.02.2001)

Author's address:

Dept. of Mathematics

University of Trento

38050 Povo (TN)

Italy

E-mail: ballico@science.unitn.it 